[1] Gestalt und Gestaltung 12

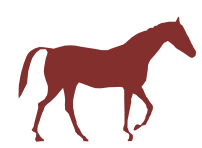

[2] Entwerfen und Entwurf 26

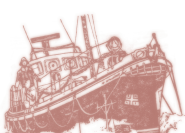

[3] Raum - Ort - Weg
Über Entwürfe reden - ein paar Einleitungen

In Gestalt von Landschaft

Punkt-Linie-Fläche-Körper

Ordnung

Form und Gestalt

Zusammenhang und Vorerfahrung

Gestalt

Superierung

Gestaltkomponenten

Induktion

Gestaltung

Zusammenhang landschaftsarchitektonischer Objekte

Zwischen Kopf und Hand

Entwerfen

Der Entwurf

Intersubjektivität

Intention

Kreativität

Bifurkation

Der übliche Entwurfsweg

Arbeitsmodell zum Entwurfsprozess (Darke, Lawsen)

Weg und Ziel

Rahmenbedingungen

Zeichen und Inhalt

\subsection{Raumbildung ("Raum")}

Raum

Landschaftsarchitektonische Räume

4 Sätze der landschaftsarchitektonischen Raumbildung

Der "reine" Raum

Die Zer-Störung des "reinen" Raumes

Raumandeutungen

Raumfolgen-Raumabstufungen

Von geschlossenen zu offenen Räumen

Raumgrenzen

Flächeneinheitlichkeit

Raumgröße in Abhängigkeit von menschlicher Nähe

Raum und Raumwirkung

Das menschliche Sehfeld

Raumwirkung und Pflanzen

Raumbildung durch Höhenunterschiede

Höhenunterschiede und Raumwirkung

Pflanzung zur optischen Reliefänderung

Der Hain

\subsection{Schwerpunktsetzung ("0rt”)}

Schwerpunkt

Besondere Lage

Die Gerade und der rechte Winkel

Betonte (gestalterische) Schwerpunkte 
3.3 Bewegung und Erschließung ("Weg")

Bewegung - Motiv und Reaktion Vorausschauende Orientierung Der "unaufmerksame” Auftritt

"Trampelpfad" - der Archetyp des Weges Positive Lenkung

Äußere und innere Erschließung Aufgaben/Ziele der inneren Erschließung Weg und Ziel

Wegverlauf und Sichtbeziehungen Wegzeichen und Wegmarken Bäume als Wegmarken Kolonnaden und Arkaden Weggelenke Die (Sitz-)Bank - Archetyp des Ver-haltens Wegenetz

Wegführung und Flächennutzung Wegführung und Raumform Weg und Raumfolgen

\subsection{Grundlagen guter Gestal} Gestalt und Zusammenhang Einheitlichkeit durch Gemeinsamkeit Gemeinsamkeit der Lage Gemeinsamkeit im Erscheinungsbild Ideell/thematische Gemeinsamkeit Unterschiedlichkeit

Reichhaltigkeit - Die Störung der Einheitlichkeit

4.2 Merkmale guter Gestalt Anregung/Unsicherheit Spannung Gewichtigkeit/Ausgewogenheit Harmonie

Verbindende Idee/Thema/Konzept Deutlichkeit

Einfachheit

4.3 Werkzeug Wiederholung Wiederholung Struktur Muster Raster Variation Transformation Rhythmus Proportion Maßstäblichkeit Zeichen

\section{[4] Gestaltqualitäten}
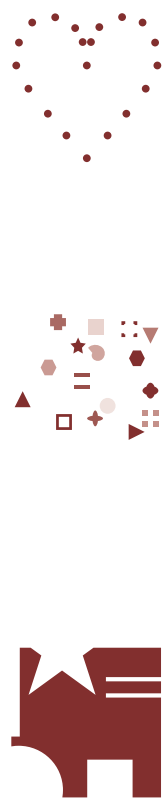

188 